\title{
Nonlinear second sound in solids
}

\author{
G. M. Tarkenton \\ Institute for Advanced Physics, 8406 Red Willow Drive, Austin, Texas 78736 \\ and Department of Physics, University of Texas at Austin, Austin, Texas 78712 \\ M. S. Cramer \\ Engineering Science and Mechanics Department, Virginia Polytechnic Institute and State University, Blacksburg, Virginia 24061
}

(Received 16 July 1993)

\begin{abstract}
We study the nonlinear propagation of thermal waves in rigid heat conductors using a model introduced by Ruggeri et al. We derive the lowest-order nonlinear corrections to the wave speed and show that the quadratic nonlinearity can be small for $\mathrm{NaF}$ and $\mathrm{Bi}$. The smallness of the nonlinear steepening parameter complicates the wave dynamics, allowing for both forward and backward steepening waves within the same pulse. We show that this mixed condition places limits on the size of admissible discontinuities; we then argue that this limit on the size of shock waves limits the arrival times of thermal signals. Using this, we propose an experiment to measure arrival times to look for this limiting behavior.
\end{abstract}

Ruggeri, Muracchini, and Seccia, ${ }^{1}$ Morro and Ruggeri $^{2,3}$ and Ruggeri ${ }^{4}$ have proposed the following set of conservation laws to describe high-frequency heat flow in rigid conductors:

$$
\begin{aligned}
& \rho \frac{\partial e}{\partial t}+\nabla \cdot \mathrm{q}=0, \\
& \frac{\partial(\alpha \mathbf{q})}{\partial t}+\nabla v=-\left(\frac{v^{\prime}}{\kappa}\right) \mathbf{q},
\end{aligned}
$$

where $\rho$ is the mass density, $e$ the internal energy, $q$ the heat flux vector, and $\alpha, v$ constitutive scalars depending on the absolute temperature, $\theta$. The quantity $\kappa$ is the (possibly) temperature-dependent thermal conductivity, and a prime indicates a derivative with respect to $\theta$. The first equation represents the first law of thermodynamics, while the second is a generalized version of the MaxwellCattaneo relation. Equation (2) reduces to Fourier's law in which the heat flux is proportional to the temperature gradient if we take the Morro-Ruggeri inertia parameter $\alpha=0$. We recover the well-known Maxwell-Cattaneo law by assuming the thermal inertia parameter is constant, and then the quantity $\tau=\alpha \kappa / \nu^{\prime}$ would be the MaxwellCattaneo relaxation time. In general, Eqs. (1) and (2) form a closed system for the fields $\theta(\mathrm{x}, t)$ and $\mathrm{q}(\mathrm{x}, t)$, once we specify the constitutive functions $e(\theta), \kappa(\theta)$, and $U_{E}(\theta)$. Here, $U_{E}(\theta)$ is the signal speed in the equilibrium limit. Ruggeri, Muracchini, and Seccia ${ }^{1}$ give a concise summary of how to determine $\alpha(\theta)$ and $v(\theta)$ from $e$ and $U_{E}$; they find that

$$
\alpha=\frac{\text { const }}{U_{E} \theta \sqrt{\rho c}}
$$

and

$$
v=\text { const } \int \frac{U_{E}(\theta)}{\theta} \sqrt{\rho c(\theta)} d \theta,
$$

where $c=e^{\prime}(\theta)$ is the specific heat.
The above references to Ruggeri and his co-workers discuss the differences between (1) and (2) and the conventional Maxwell-Cattaneo theory. In particular, they show that the generalized Fourier law (2) satisfies all commonly accepted physical principles, including the expectation that the system, (1) and (2), be hyperbolic and that the specific heat, $c$, remain non-negative for all $\theta>0$. In the linear limit, both the Maxwell-Cattaneo and Morro-Ruggeri theories yield precisely the same results. Thus, the most convenient way to distinguish between these macroscopic models is through the use of experiments or theories which involve nonlinear effects. The purpose of this work is to outline a theory of weakly nonlinear, weakly dissipative heat waves based on (1) and (2). Our restriction to weakly dissipative configurations comes from the fact that frequencies and wavelengths corresponding to strong dissipation tend to obscure the nonlinear effects of interest here. The simplification to weakly nonlinear systems will reveal the underlying dynamics with a minimum of complication. Furthermore, this assumption does not appear to be overly restrictive given the amplitudes attained in recent experiments (e.g., Jackson, Walker, and McNelly, ${ }^{5}$ McNelly et al., ${ }^{6}$ Jackson and Walker, ${ }^{7}$ Narayanamurti and Dynes ${ }^{8}$ ).

We now focus our attention on one-dimensional $[\theta=\theta(x, t), q=q(x, t)]$ simple waves propagating in an initially uniform equilibrium $\left\{(\theta, q)=\left(\theta_{0}, 0\right)\right\}$ undisturbed state. Here, $t$ is the time and the positive $x$ direction lies along the direction of propagation. The subscript 0 will always denote properties evaluated at the undisturbed state. We assume that both the nonlinear distortion and the decay due to a finite conductivity are small; the decay rate is assumed to be on the same order of magnitude as the nonlinear distortion or smaller. The authors have applied both the systematic perturbation scheme developed by Taniuti and $\mathrm{Wei}^{9}$ and a firstprinciples approximation based on the method of characteristics to the hyperbolic system (1) and (2). In either case, we found the resulting nonlinear wave speed to be 


$$
\lambda=U_{E 0}\left\{1+\Gamma_{0} \theta_{0}\left(\frac{\theta-\theta_{0}}{\theta_{0}}\right)+O\left(\frac{\theta-\theta_{0}}{\theta_{0}}\right)^{2}\right\},
$$

where

$$
\Gamma(\theta)=\frac{1}{2} \frac{\alpha}{U_{E}^{2}}\left(\frac{U_{E}^{2}}{\alpha}\right)^{\prime}=\frac{3}{2} \frac{U_{E}^{\prime}}{U_{E}}+\frac{1}{2} \frac{c^{\prime}}{c}+\frac{1}{2 \theta}
$$

and $U_{E 0}=U_{E}\left(\theta_{0}\right)$. In (5) we have used the first of (3) to obtain the latter form of the nonlinear steepening parameter.

In order to obtain numerical values for the steepening parameter (5), we follow Ruggeri, Muracchini, and Sec$\mathrm{Cia}^{1}$ and Ruggeri ${ }^{4}$ in using the Debye specific heat and Coleman and Newman's ${ }^{10}$ fit for the equilibrium wave speed, i.e.,

$$
c=\text { const } \cdot \theta^{3}
$$

and

$$
U_{E}(\theta)=\left(A+B \theta^{n}\right)^{-2},
$$

where $A, B$, and $n$ are empirical constants. The resultant expression for $\Gamma(\theta)$ is

$$
\theta \Gamma=\frac{4}{5} \frac{1+\left(1-\frac{3}{5} n\right)(B / A) \theta^{n}}{1+(B / A) \theta^{n}} .
$$

We plot the nondimensional combination $\theta \Gamma$ in Fig. 1 for the materials $\mathrm{NaF}$ and $\mathrm{Bi}$, obtained using the parameters given by Ruggeri, Muracchini, and Seccia ${ }^{1}$ and Ruggeri. ${ }^{4}$ This figure shows that $\Gamma=0$ for each of these substances in the temperature ranges studied. The temperature where $\Gamma$ vanishes is $\theta_{\text {critical }}=[(A / B) 5 /(3 n-5)]^{1 / n}$, and corresponds exactly to $\widetilde{\theta}$ in Ruggeri. ${ }^{4}$

The fact that the steepening parameter $\Gamma$ changes sign indicates that at least two qualitatively different evolutions are possible. These different evolutions are summarized in Fig. 2, where we show the temperature distributions corresponding to $\Gamma_{0}>0$ and $\Gamma_{0}<0$ undisturbed states. When $\Gamma_{0}>0$, the warmer regions of the wave

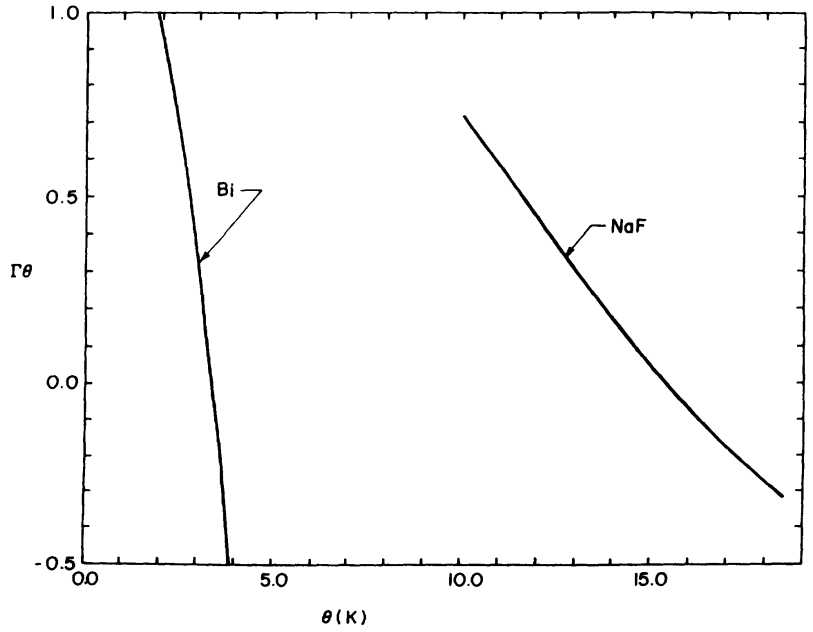

FIG. 1. Plot of nondimensional steepening parameter as a function of temperature for $\mathrm{Bi}$ and $\mathrm{NaF}$. propagate faster than the colder regions, and the wave tends to steepen forward, forming the temperatureraising shock sketched in Fig. 2(a). On the other hand, if $\Gamma_{0}<0$, the warmer regions tend to move slower than the cooler regions, and the steepening is backwards, relative to the propagation direction, as depicted in Fig. 2(c). Similar observations were made in the context of second sound in liquid He II; see, for example, Putterman. ${ }^{11}$ The apparent interpretation of the so-called "critical" temperature is that the nonlinear steepening is identically zero. That is, simple wave solutions to the nonlinear system (1) and (2) propagate linearly.

Because the higher-order terms correctly neglected in the $\theta_{0} \Gamma_{0}=\boldsymbol{O}(1)$ theory may be as large as the lowestorder nonlinearity retained in (4), we expect that the approximation scheme leading to (4) is invalid when $\Gamma_{0} \theta_{0}$ is sufficiently small. A more physical argument leading to the conclusion that $(4)$ is incorrect in the vicinity of $\Gamma \approx 0$ is that any finite strength wave having $\theta_{0} \Gamma_{0}$ small and positive is likely to result in a change in sign of the local value of the steepening parameter. As a result, simultaneous backward and forward steepening within the same pulse is possible. Because $\theta_{0} \Gamma_{0}$ is a numerical constant in (4), such a sign change in the local value of $\Gamma$ is not consistent with (4). Thus, we need to amend the approximation to the wave speed (4) to incorporate the additional assumption of small $\theta_{0} \Gamma_{0}$.

A self-consistent approximation valid in the vicinity of the zeros of $\Gamma$ seen in Fig. 1 follows from the assumptions
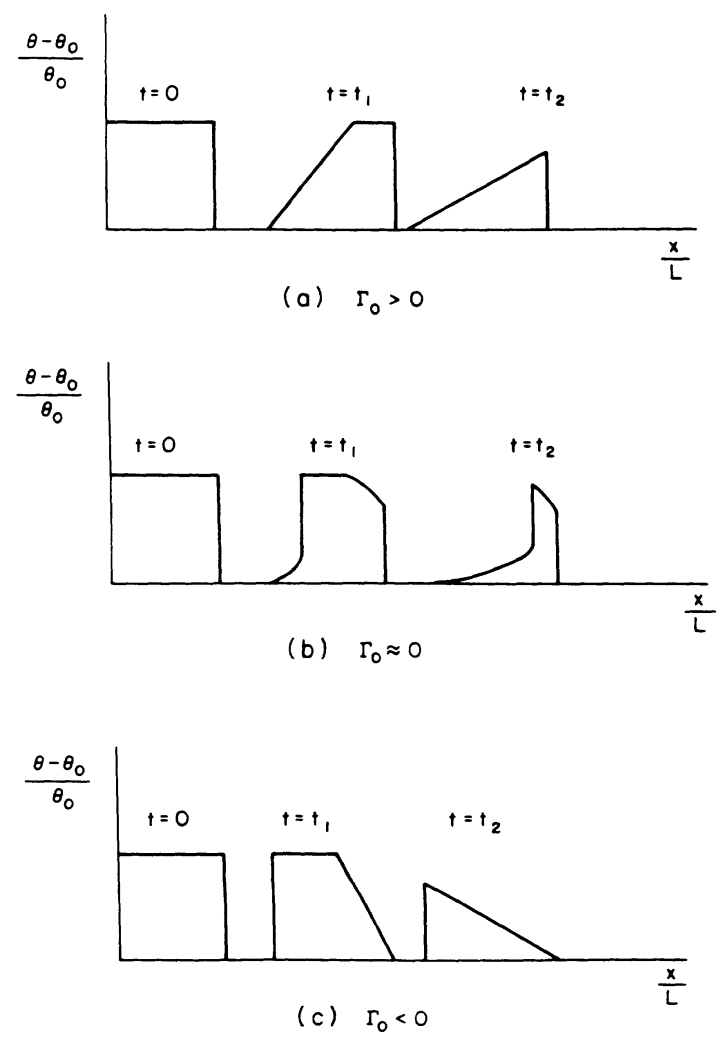

FIG. 2. Sketch of the evolution of a square-wave initial pulse. (a) $\Gamma_{0} \theta_{0}=O(1)$ and positive, (b) $\Gamma_{0} \theta_{0}$ small and positive, and (c) $\Gamma_{0} \theta_{0}=O(1)$ and negative. 


$$
\theta_{0} \Gamma_{0}=O\left(\frac{\theta-\theta_{0}}{\theta_{0}}\right)=O\left[\frac{L}{L_{d}}\right]^{1 / 2}=o(1)
$$

where $L_{d} \equiv \kappa_{0} /\left(U_{E 0} \rho c_{0}\right)$ is the skin depth and the $L$ is the wavelength. As expected, we take the nondimensional measure of $\Gamma_{0}$ to be small, in addition to the usual small disturbance and weak dissipation $\left[L=o\left(L_{d}\right)\right]$ assumptions. The relation between $\theta_{0} \Gamma_{0}$ and the wave amplitude allows for the possibility that part of the wave can correspond to positive $\Gamma$ (forward steepening) while other parts may correspond to negative values of $\Gamma$ (backward steepening). The condition relating the wavelength to the wave amplitude ensures that the typical decay time $\tau_{d}=O\left(L_{d} / U_{E 0}\right)$ is no shorter than the time over which significant nonlinear steepening may be observed; the latter time scale is of order

$$
\frac{L}{U_{E 0}} \frac{1}{\theta_{0} \Gamma_{0}\left[\left(\theta-\theta_{0}\right) / \theta_{0}\right]}=O\left[\frac{L}{U_{E 0}}\left(\frac{\theta-\theta_{0}}{\theta_{0}}\right]^{-2}\right] \text {. }
$$

Finally, we confine our attention to simple right-running waves and seek an approximation that is valid over time scales on the order of those corresponding to nonlinear steepening; both of these general assumptions were also implicit in the derivation of (4) and (5).

Under the assumptions discussed above, two approaches can be employed to derive the appropriate approximation to (1) and (2). In the first, one could simply solve the hyperbolic system (1) and (2) with the method of characteristics and then approximate the resultant solution in a manner consistent with our assumptions; this approach is essentially the same as the first-principles method of characteristics approach used to derive (4) and (5). A second equivalent approach is to apply the systematic perturbation scheme developed by Cramer and $\operatorname{Sen}^{12}$ to (1) and (2). This scheme was specifically designed to extend the method of Taniuti and $\mathrm{Wei}^{9}$ to cases where the quadratic steepening parameter is small. As a check on the algebra, we applied both techniques independently and demonstrated the expected consistency between the two approaches. The result of both approaches is that the wave evolution is governed by the following modification of the inviscid Burgers equation:

$$
\frac{\partial u}{\partial \bar{t}}+\frac{\lambda}{U_{E 0}} \frac{\partial u}{\partial \bar{x}}=-\frac{\delta}{2} u
$$

where $\bar{x}=x / L, \bar{t}=t U_{E 0} / L$ are the nondimensional forms of the spatial and temporal variables, $\delta=L / L_{d} \ll<1$ is a nondimensional dissipation parameter,

$$
u \equiv \frac{\theta-\theta_{0}}{\theta_{0}} \approx \frac{q}{\rho c_{0} U_{E 0} \theta_{0}}
$$

is a nondimensional measure of the disturbance function, and

$$
\lambda=U_{E 0}\left\{1+\Gamma \theta_{0} u+\frac{1}{2} \Lambda \theta_{0}^{2} u^{2}\right\}
$$

is the correct approximation to the nonlinear wave speed when $\theta_{0} \Gamma_{0}$ is small. The new nonlinearity parameter, $\Lambda$, has the following form:

$$
\begin{aligned}
\Lambda & \equiv \frac{U_{E}^{\prime \prime}}{U_{E}}-\frac{\alpha^{\prime \prime}}{\alpha}+\frac{1}{2} \frac{\alpha^{\prime}}{\alpha} \frac{c^{\prime}}{c}+\frac{5}{4}\left(\frac{\alpha^{\prime}}{\alpha}\right)^{2} \\
& =2 \frac{U_{E}^{\prime \prime}}{U_{E}}+\frac{1}{2} \frac{c^{\prime \prime}}{c}-\frac{2}{3}\left(\frac{c^{\prime}}{c}\right)^{2}-\frac{1}{\theta^{2}}-\frac{1}{3 \theta} \frac{c^{\prime}}{c}
\end{aligned}
$$

with all derivative evaluated as the undisturbed temperature $\theta_{0}$. To obtain the form (13a), one must explicitly use the condition that $\theta_{0} \Gamma_{0}$ is small. Form (13b) follows from (13a) upon using the relation (3). As discussed by Cramer and Sen, ${ }^{12}$ (13) may be evaluated at either the actual undisturbed state or the zero of $\Gamma$, at least to the accuracy implicit in (10)-(13).

We can use (6) to determine numerical values for the cubic nonlinearity parameter. If, as discussed above, we evaluate $\Lambda$ at the zero of $\Gamma$ for each conductor, we find that $\Lambda \theta_{\text {critical }}^{2}=-4.333$ for $\mathrm{NaF}$ and $\Lambda \theta_{\text {critical }}^{2}=-5.423$ for $\mathrm{Bi}$. We intuit the fact that $\Lambda<0$, since $(\theta \Gamma)^{\prime}<0$ at the zero of $\Gamma$. However, it is important to note that $\Lambda \neq(\theta \Gamma)^{\prime} / \theta$ or any other simple form of $\Gamma^{\prime}$. The assumption that $\Lambda$ should be proportional to $\Gamma^{\prime}$ is usually based on the idea that the cubic nonlinearity arises directly from the quadratic nonlinearity through the use of a Taylor series. As discussed by Cramer and Sen, ${ }^{13,12}$ the approximations leading to the lowest-order quadratic steepening parameter may neglect terms which are important when the cubic nonlinearity is computed. Furthermore, the calculation of the cubic nonlinearity necessarily involves the first-order disturbances (the linear theory being zeroth order) which are correctly ignored in theories restricted to the study of the quadratic nonlinearity.

Having obtained the correct nonlinear wave speed (12), we should briefly address the issue of shock structure. Here, we follow Olenick ${ }^{14}$ and Lax $^{15}$ in writing the shock speed corresponding to (10) as

$\lambda_{s}=U_{E 0}\left\{1+\Gamma_{0} \theta_{0} \frac{u_{1}+u_{2}}{2}+\Lambda \theta_{0}^{2} \frac{u_{1}^{2}+u_{1} u_{2}+u_{2}^{2}}{6}\right\}$,

where subscripts 1 and 2 denote conditions immediately ahead of and behind the shock, respectively. We will also follow the above authors in employing the speed-ordering conditions

$$
\lambda_{2} \geq \lambda_{s}>\lambda_{1}
$$

in order to distinguish admissible from inadmissible shocks. If we assume that the present model can be embedded in a more comprehensive diffusive, rather than dispersive, theory, i.e., that we replace (15) by a "viscosity criterion" (cf. Hagan and Slemrod ${ }^{16}$ ) to determine shock existence, then it is easy to show that (15) is both necessary and sufficient for the existence of shock waves in the cubic theory presented here.

The equality in (15) corresponds to shock waves which propagate at the characteristic speed of the state immediately behind the shock. Such states are similar to Chapman-Jouget points in detonation wave theory and are commonly referred to as sonic shocks in nonlinear 
gas dynamics (see, e.g., Cramer and Kluwick ${ }^{17}$ or the articles by Cramer $^{18}$ or Kluwick ${ }^{19}$ ). Because the present context involves thermal rather than sound waves, we will refer to shocks with $\lambda_{s}=\lambda_{2}$ as thermic shocks. We should also note here that the class of shocks $\lambda_{s}=\lambda_{1}$ is expected to be inadmissible when $\Lambda<0$. From this, we expect such upstream thermic shocks to appear only when $\Lambda>0$.

As pointed out be Ruggeri, Muracchini, and Seccia, ${ }^{1}$ the dynamics of the present model can be considerably more complex than those where the steepening is strictly positive or strictly negative. A configuration typical of the $\Gamma_{0} \theta_{0} \approx 0$ theory is sketched in Fig. 2(b) for $\Gamma_{0}>0$, $\Lambda<0$ and a square-wave input. The new feature characteristic of the present theory is the fact that both temperature-lowering and temperature-raising shocks appear in the same pulse. Such double-shock configurations have been observed experimentally in the related area of second sound in $\mathrm{He}$ II by Turner ${ }^{20}$ and Torczynski. ${ }^{21}$ Cramer and Sen ${ }^{13}$ present detailed solutions to the $\delta=0$ version of (10) and (11) and (14) and (15); because of the canonical nature of these solutions, the He II work can be carried over to the $\delta=0$ version of the present study with a minimum of complication.

Previous investigations have revealed that thermic shocks will frequently occur when an inadmissible discontinuity is introduced through initial or boundary conditions. In particular, the inadmissible discontinuity will break up into either a centered temperature-raising or temperature-lowering fan or a thermic shock-centered fan combination. Examples in the context of gas dynamics can be found in the work of Wendroff ${ }^{22}$ and Cramer and Kluwick. ${ }^{17}$

We can use this information to design an experiment to observe some of these nonlinear effects. We envision a one-dimensional crystal at an equilibrium temperature, $\theta_{0}$, with a constant heat flux $Q_{0}$ located at the origin with a detector a distance $H$ to the right. In the context of the $\delta=0$ theory, we can easily predict the travel time across the sample for this pulse. (The $\delta \neq 0$ case is considerably more involved and we will present detailed solutions at a later time.) If the magnitude, $u_{0}=Q_{0} /\left(\rho c_{0} U_{E 0} \theta_{0}\right)$, is below the thermic shock value of $3 \Gamma_{0} / 2 \theta_{0}|\Lambda|$, then the heat pulse propagates as a conventional temperatureraising shock wave with speed (14) with $u_{1}=0$ and $u_{2}=u_{0}$. The arrival time at the detector will be

$$
t_{\mathrm{arr}}=\frac{H}{U_{E 0}}\left[1-\frac{\Gamma_{0} \theta_{0}}{2} u_{0}+\frac{|\Lambda| \theta_{0}^{2}}{6} u_{0}^{2}\right),
$$

which we recast as

$$
\Delta=\frac{A}{2}\left[1-\frac{A}{3}\right]
$$

where

$$
\Delta=\frac{|\Lambda|}{\Gamma_{0}^{2}}\left(1-\frac{U_{E 0} t_{\mathrm{arr}}}{H}\right)
$$

is the scaled arrival time deviation, which encodes the

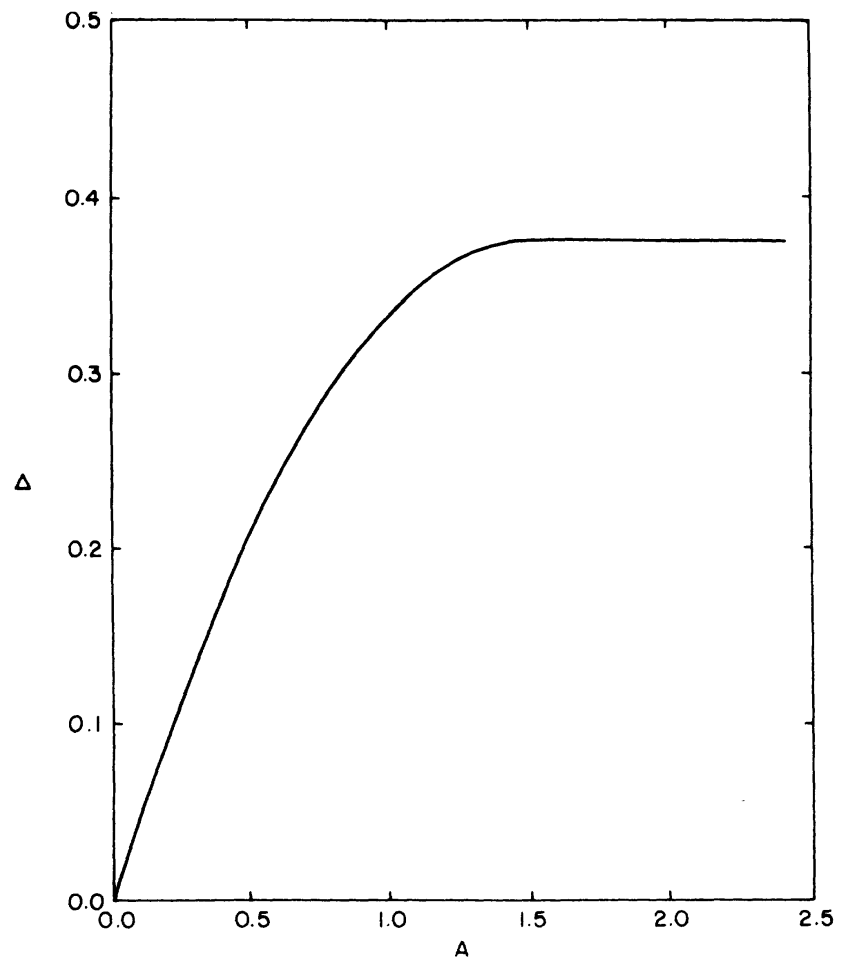

FIG. 3. Plot of arrival time deviation, $\Delta$ [Eq. (17)], as a function of scaled amplitude, $A$.

nonlinear character of the wave, and $A=u_{0}|\Lambda| \theta_{0} / \Gamma_{0}$ is the scaled amplitude. If the initial pulse strength is larger than $A=\frac{3}{2}$, then the discontinuity is inadmissible and it breaks up into a fan-thermic shock configuration. This implies that the arrival times should saturate at the arrival time for the thermic shock: $\Delta=\frac{3}{8}$. Figure 3 shows the complete behavior of the arrival times for any amplitude. The definitive signature of nonlinear behavior is the leveling off of the arrival time with increasing amplitude.

To see if these effects are detectable, we calculate the difference between the linear and nonlinear arrival times of a thermic disturbance for $\mathrm{NaF}$ at $\theta_{0}=12 \mathrm{~K}$ and $\mathrm{Bi}$ at $\theta_{0}=2.5 \mathrm{~K}$. In each case, the numerical values for $\Lambda \theta_{0}^{2}$ are those given above. The remaining parameters are summarized in Table $I$. Then the deviation is simply $3 \Gamma_{0}^{2} / 8|\Lambda|$, which is 0.016 for $\mathrm{NaF}$ and is 0.031 for $\mathrm{Bi}$. Thus, a sample crystal with a length $H=1 \mathrm{~cm}$ requires a temporal resolution of at least $61 \mathrm{~ns}$ for $\mathrm{NaF}$ and $331 \mathrm{~ns}$ for $\mathrm{Bi}$, within the reach of $100-\mathrm{MHz}$ transducers.

We have derived the lowest-order nonlinear corrections to the wave speed for thermal waves (second sound)

TABLE I. This table shows the values of $\theta \Gamma$ and $U_{E}$ for $\mathrm{NaF}$ and $\mathrm{Bi}$ for the conditions of the experiment outlined in the text.

\begin{tabular}{ccc}
\hline \hline & $\theta_{0} \Gamma_{0}$ & $\begin{array}{c}U_{E 0} \\
(\mathrm{~cm} / \mathrm{s})\end{array}$ \\
\hline $\mathrm{NaF}$ & 0.434 & $2.67 \times 10^{5}$ \\
$\mathrm{Bi}$ & 0.670 & $9.36 \times 10^{4}$ \\
\hline
\end{tabular}


in solids using the constitutive theory of Ruggeri, Muracchini, and Seccia ${ }^{1}$ and Ruggeri ${ }^{4}$ for the region where the quadratic nonlinearity is small. We identified the temperature at which the nonlinearity parameter changes sign with Ruggeri's critical temperature and outlined the ex- istence theory for discontinuities within this constitutive model. Finally, we have proposed an experiment to measure the arrival times of heat pulses to demonstrate some of the nonlinear effects predicted with this model.
${ }^{1}$ T. Ruggeri, A. Muracchini, and L. Seccia, Phys. Rev. Lett. 64, 2640 (1990).

${ }^{2}$ A. Morro and T. Ruggeri, Int. J. Non-Linear Mech. 22, 27 (1987).

${ }^{3}$ A. Morro and T. Ruggeri, J. Phys. C. 21, 1743 (1988).

${ }^{4}$ T. Ruggeri, Le Mathematiche 46, 379 (1991).

${ }^{5}$ H. Jackson, C. Walker, and T. McNelly, Phys. Rev. Lett. 25, 26 (1970).

${ }^{6}$ T. McNelly et al., Phys. Rev. Lett. 24, 100 (1970)

${ }^{7}$ H. Jackson and C. Walker, Phys. Rev. B 3, 1428 (1971).

${ }^{8}$ V. Narayanamurti and R. Dynes, Phys. Rev. Lett. 28, 1461 (1972).

${ }^{9}$ T. Taniuti and C.-C. Wei, J. Phys. Soc. Jpn. 24, 941 (1968).

${ }^{10}$ B. Coleman and D. Newman, Phys. Rev. B 37, 41492 (1988).

${ }^{11}$ S. J. Putterman, Superfluid Hydrodynamics (North-Holland, Amsterdam, 1974).
${ }^{12}$ M. Cramer and R. Sen, J. Fluid Mech. 221, 233 (1990).

${ }^{13}$ M. Cramer and R. Sen, J. Wave Motion 15, 333 (1992).

${ }^{14}$ G. Olenick, Am. Math. Soc. Transl. (Sec. 2) 33, 285 (1963).

${ }^{15}$ P. D. Lax, in Contributions to Nonlinear Functional Analysis, edited by E. H. Zarantello (Academic, New York, 1971).

${ }^{16}$ R. Hagan and M. Slemrod, Arc. Rational Mech. Anal. 83, 333 (1983).

${ }^{17}$ M. Cramer and A. Kluwick, J. Fluid Mech. 142, 9 (1984).

${ }^{18} \mathrm{M}$. Cramer, in Nonlinear Waves in Real Fluids, edited by A. Kluwick (Springer-Verlag, New York, 1991).

${ }^{19}$ A. Kluwick, in Nonlinear Waves in Real Fluids, edited by A. Kluwick (Springer-Verlag, New York, 1991).

${ }^{20}$ T. Turner, Physica B 107, 701 (1981).

${ }^{21}$ J. Torczynski, D. Gerthsen, and T. Roesgen, Phys. Fluids 27, 2418 (1984).

${ }^{22}$ B. Wendroff, J. Math. Anal. Appl. 38, 454 (1972). 